Running head: Partners of breast cancer patients

\title{
Psychosocial adjustment and marital intimacy among partners of breast cancer patients: A comparison study with partners of healthy women
}

\author{
Helena Moreira \& Maria Cristina Canavarro
}

Faculty of Psychology and Educational Sciences, University of Coimbra, Coimbra, Portugal

Correspondence concerning this article should be addressed to:

Helena Moreira, Research Group “Relações, Desenvolvimento e Saúde”, Faculdade de Psicologia e Ciências da Educação, Universidade de Coimbra, Rua do Colégio Novo, Apartado 6153, 3001-802 Coimbra, Portugal.

E-mail: hmoreira@,fpce.uc.pt

Cite as:

Moreira, H., \& Canavarro, M. C. (2013). Psychosocial adjustment and marital intimacy among partners of breast cancer patients: A comparison study with partners of healthy women. Journal of Psychosocial Oncology, 31, 282-304. doi:10.1080/07347332.2013.778934. 


\begin{abstract}
This cross-sectional study investigated the psychosocial adjustment of 70 partners of breast cancer patients by comparing their emotional adjustment and quality of life (QoL) with 70 partners of women without cancer. The role of marital intimacy in their adjustment and the moderating effect of group type were analyzed. The partners of breast cancer patients presented higher levels of anxious and depressive symptomatology; poor social, psychological, general and physical QoL; and higher levels of intimacy. The moderation analysis showed that higher levels of intimacy predicted lower levels of depression and anxiety and greater QoL only among the partners of cancer patients.
\end{abstract}

\title{
Key-words
}

Breast cancer; partner; psychosocial adjustment; marital intimacy; quality of life; emotional adjustment 


\section{Introduction}

The development of a disease in a family member may influence an entire family (Broderick, 1993). Therefore, breast cancer can be viewed as a disease that has a potentially significant effect on all family members (Kim \& Given, 2008; Manne \& Badr, 2008) and, in particular, on a woman's partner (Carlson, Bultz, Speca, \& Pierre, 2000; Dorros, Card, Segrin, \& Badger, 2010). Nevertheless, research has primarily focused on the individual adjustment of women to the disease. Psycho-oncology researchers have begun to emphasize the need to analyze this disease within the family context and specifically in the context of marital relationships only in the past two decades (Braun, Mikulincer, Rydall, Walsh, \& Rodin, 2007; Carlson et al., 2000; Manne \& Badr, 2008; O'Mahoney \& Carroll, 1997). Since this research emerged, several studies have examined the influence of cancer on marital relationships (e.g., Dorval at al., 2005; Hegerdoon, Sanderman, Coyne, Bolks, \& Tuinstra, 2008) and have thus enabled a deeper and more comprehensive knowledge regarding the functioning of couples in this context (Hegerdoon et al., 2008; Manne \& Badr, 2008; O’Mahoney \& Carroll, 1997).

However, much less is known about the psychosocial adjustment of partners of breast cancer patients and the influence of marital relationships on their adjustment. Therefore, the current study intends to investigate the psychosocial adjustment of the partners of breast cancer patients by comparing their emotional adjustment and quality of life (QoL) with a group of partners of women without any history of cancer. In addition, this study intends to analyze the role of marital intimacy in the adjustment of partners.

\section{The Psychosocial Adjustment of Partners}

Throughout the course of the disease, the partners of breast cancer patients confront a number of challenges and demands, such as coping with the anxiety that is related to the potential negative outcomes of cancer, assisting their spouses in coping with negative 
emotions, and managing the practical and role changes that result from the disease (Kayser \& Scott, 2008). In addition, partners are typically the main informal caregivers of such patients. The demands that are associated with the provision of care can significantly interfere with a partner's life and routines (Nijboer et al., 1998). For instance, caregivers must often assume many of the traditional female roles (e.g., managing a household and performing childcare responsibilities) and perform a number of caregiving tasks, such as providing emotional support (e.g., listening to, empathizing with, reassuring and comforting the patient) and instrumental support (e.g., accompanying the patient to treatment and medical appointments and performing household tasks). Consequently, such partners may sacrifice their own gratification activities, such as social, leisure or family activities, to assist patients (Manne, Alfieri, Taylor, \& Dougherty, 1999). All of these responsibilities can become burdensome and significantly affect the physical and psychological health of partners. The caregiver burden (that is, the experience of persistent stress or negative experiences that result from the provision of care to a patient) has proven to be relatively common (Kim \& Given, 2008; Nijboer et al., 1998).

Given these challenges and difficulties, breast cancer is expected to have a considerable effect on the emotional adjustment and QoL of partners. Nevertheless, this issue has rarely been investigated and has primarily been addressed by comparing the psychosocial adjustment of partners with that of female patients. Given the interdependence that characterizes a couple, one would expect a mutual influence and similar levels of disturbance between a woman and her partner. Indeed, some studies have found similar rates of psychological distress across partners that often remain throughout the disease course. For instance, Northouse, Templin, Mood, and Oberst (1998) observed a high degree of correspondence between patients with breast cancer and their partners on several adjustment measures over three evaluation periods (diagnosis period, 60-day period and the period one 
year after diagnosis). The authors found that $56 \%$ of the couples who had been classified in the "high" disturbance group at the time of diagnosis retained their classification after 60 days; $50 \%$ of the couples retained this classification one year later. In a subsequent study, Northouse, Templin, and Mood (2001) found moderately high correlations between patients and partners for several measures of adjustment throughout all 12 months of the study. Similarly, Segrin, Badger, Dorros, Meek, and Lopez (2007) observed a strong and positive correlation between the levels of anxiety of patients and that of their partners during a 10week longitudinal study.

Few studies have compared the adjustment outcomes of this specific group of men with a group of men from the general population (i.e., spouses of women with no history of cancer). For instance, Wagner, Bigatti, and Storniolo (2006) compared the QoL of a group of men whose wives were healthy with a group of men whose wives had breast cancer and found that the QoL of the latter group was significantly lower. Specifically, the men whose wives had breast cancer received lower scores on the role-emotional, general health, vitality, and mental health subscales of the SF-36 health survey. In fact, the use of a control group to investigate the adjustment of partners is of paramount importance, as this approach would enable the examination of the extent to which partners depart from the norm and the influence of cancer on their lives.

More recently, some population-based studies on the psychiatric effects of cancer on partners have been conducted. For instance, Nakaya et al. (2010) examined the risk of severe depression in 20,538 partners of women with breast cancer and identified an increased risk of hospitalization with an affective disorder among this group of men compared with the partners of healthy women. Another study (Sjövall et al., 2009), which was based on a sample of 11,076 partners of cancer patients, also showed an increase in the likelihood that partners 
would receive a psychiatric diagnosis after the diagnosis of this disease. These recent studies clearly demonstrate the negative influence of cancer on the mental health of partners.

Although the characterization of the psychosocial adjustment of partners and the comparison with a control group are extremely important, it is equally relevant to analyze the factors that may influence their adjustment. In this study, we will focus on marital intimacy.

\section{The Role of Marital Intimacy in the Adjustment of Partners}

Research has shown that intimacy is related to several positive aspects of a relationship, such as greater marital satisfaction (Greeff \& Malherbe, 2001; Prager, 1995) and improvements in the psychological and physical health of each element of the dyad (Prager, 1995; Prager \& Buhrmester, 1998). Prager (1995) argued that intimate relationships buffer the pathogenic effects of stress and provide several mental and physical health benefits.

Currently, the clearly beneficial effects of intimacy on individual health and well-being are widely accepted.

Intimacy is often considered a primary psychological need (Manne \& Badr, 2008; Prager, 1995) and a crucial characteristic of most marital relationships (Broucke, Vandereycken, \& Vertommen, 1995). This construct has been extensively investigated in psychology, and many definitions and conceptualizations have been proposed (e.g., Laurenceau, Barrett, \& Pietromonaco, 2004; Prager, 1995; Reis \& Patrick, 1996; Reis \& Shaver, 1988; Schaefer \& Olson, 1981). Within the psycho-oncology field, Manne and Badr (2008) developed the relationship intimacy model of couple psychosocial adaptation to cancer, whereby intimacy is defined as "the experience of feeling close to and cared for by a partner with regard to the cancer experience" (p. 2548). This model is based on the interpersonal process model of intimacy (Reis \& Patrick, 1996; Reis \& Shaver, 1988), according to which intimacy is an interpersonal and transactional process whereby an individual communicates personally relevant and revealing information, thoughts and feelings 
to another person and, as a result of the responsiveness of the other person, feels validated, cared for and understood.

According to Manne and Badr's model, higher levels of intimacy lead to higherquality relationships and enhanced psychological adjustment in both spouses. However, few studies have attested to the important role of intimacy in the adjustment of patients and partners, and the majority of these studies did not focus on breast cancer (Manne \& Badr, 2010; Manne, Badr, Zaider, Nelson, \& Kissane, 2010). For instance, this association was found among head and neck and lung cancer patients (Manne \& Badr, 2010) and among those with prostate cancer (Manne et al., 2010). Moreira, Crespo, Silva, Paredes, and Canavarro (2010) found that breast cancer patients who reported greater intimacy had better social and psychological QoL during the adjuvant treatment phase.

Studies on the link between the intimacy and adjustment outcomes of partners are even scarcer. For instance, Manne and Badr (2010) found that partners of head and neck and lung patients who reported high levels of intimacy (both general and cancer-related intimacy) showed lower levels of psychological distress. To our knowledge, no study has investigated this association among partners of breast cancer patients. However, more intimate relationships may also foster better adjustment outcomes for these men. Therefore, this study intends to investigate the link between these variables among a sample of partners of breast cancer patients.

\section{Study Aims and Hypotheses}

This study intends to overcome some important gaps in the literature: the dearth of research on the psychosocial adjustment of partners of breast cancer patients, the insufficient use of a comparison group of partners of women without cancer history, and the dearth of knowledge regarding the role of marital intimacy in the psychosocial adjustment of partners of breast cancer patients. Therefore, the first goal of this study was to characterize the 
adjustment (i.e., emotional distress and QoL) of partners during the first five years following the women's diagnosis by comparing a group of spouses of breast cancer patients and a group of spouses of healthy women. In addition, according to the treatment and surgery the cancer patients had received, their spouses were divided into subgroups, thus allowing to explore the influence of the different treatment possibilities during their adaptation.

The second goal was to examine the role of marital intimacy in the adjustment of partners and to determine whether there was a moderating effect based on groups (clinical vs. comparison). That is, this study intends to examine whether the link between intimacy and adjustment is more evident among the husbands of breast cancer patients or whether the influence of intimacy on psychosocial adjustment is equally important for these partners and those of healthy women.

Based on previous research (e.g., Wagner et al., 2006), it was hypothesized that the partners of breast cancer patients would report higher levels of emotional distress and lower QoL than the husbands of healthy women. In addition, based on the model of Manne and Badr (2008), it is expected that higher levels of marital intimacy would be related to lower emotional distress and greater QoL.

\section{Method}

\section{Participants}

The sample included 140 men: 70 partners of women with breast cancer (group 1 G1) and 70 men whose wives had no history of cancer (group 2-G2). The criteria for inclusion in this study consisted of the following: (1) being married or living with a significant other for a minimum of one year, (2) no personal history of cancer, (3) no major disabling medical or psychiatric conditions, and (4) the ability to read and write Portuguese. Additionally, a primary criterion for inclusion in G1 was the wife's diagnosis of nonmetastatic breast cancer a maximum of 5 years ago. The G2 participants had comparable 
sociodemographic characteristics (age, education status and length of marriage), and their wives had not been diagnosed with an oncological disease.

The G1 partners had a mean age of 53.88 years old $(S D=9.39$; range $=30-71)$, and the length of their marriages ranged from 1 to 46 years, with a mean of 26.74 years $(S D=$ $11.98) ; 82.9 \%$ of these partners completed 12 or fewer years of schooling. Their wives had a mean age of 51.50 years old $(S D=8.40$; range $=30-68)$ and were a mean of 16.62 months $(S D=21.43 ;$ range $=1-60)$ post-diagnosis. Approximately half of the women $(52.9 \%)$ were diagnosed with invasive ductal carcinoma, and the majority (62.9\%) underwent mastectomy. In addition, $37.1 \%$ of the women underwent chemotherapy followed by radiotherapy, $22.9 \%$ underwent chemotherapy, and $40 \%$ underwent radiotherapy. When the partners participated in the study, $68.6 \%$ of the women were undergoing some type of treatment (chemotherapy or radiotherapy), and $31.4 \%$ of the women had already completed their treatments. The ages of the $\mathrm{G} 2$ participants ranged from 30 to 65 years, with a mean of 51.20 years $(S D=6.33)$. The length of their marriages ranged from 1 to 41 years, with a mean of 24.83 years $(S D=7.90)$; the majority of these participants $(78.6 \%)$ had completed 12 or fewer years of schooling. All of the couples from G1 and G2 were married.

\section{Procedures}

The partners of breast cancer patients were recruited in the gynecologic and chemotherapy departments of Coimbra University Hospitals EPE (CUH), the main public hospital in the central region of Portugal. Ethical approval was obtained from the CUH Research Ethics Committee. Partners were invited to participate in the study before the chemotherapy or radiotherapy session or during a routine appointment. The participants received a letter explaining the study and the self-report questionnaires. Most of the partners completed the assessment protocol at home and either returned it by mail via a postage-paid, pre-addressed envelope or gave it to the researcher directly. 
The group of husbands of women without cancer history was selected from a random community sample using convenience procedures. Only married men of comparable age, education status and length of marriage were included in the study. Husbands of women with a previous history of cancer were excluded. An envelope with the questionnaire package, the consent form, a letter informing potential subjects about the study and a postage-paid, preaddressed envelope for the return of materials was given to each participant.

All participants received a detailed explanation of the research objectives and confidentiality requirements and provided their informed consent before completing the questionnaire.

\section{Measures}

Emotional adjustment. The Portuguese version of the Hospital Anxiety and Depression Scale (HADS; Pais-Ribeiro et al., 2007; Zigmond \& Snaith, 1983) was used to assess current levels of depression (e.g., I feel cheerful) and anxiety (e.g., I feel tense or 'wound up'). This scale contains 14 items and uses a 4-point scale that ranges from 0 to 3 . The total score for each subscale ranges from 0 to 21 , and higher scores indicate higher levels of symptomatology. The Portuguese version of the HADS has demonstrated good psychometric qualities (Pais-Ribeiro et al., 2007). In this sample, the Cronbach's alpha coefficients were .81 (G1) and .65 (G2) for anxiety and .77 (G1) and .73 (G2) for depression.

Quality of life. To measure individual subjective perceptions of QoL, we used the World Health Organization Quality of Life-BREF instrument (WHOQOL-BREF; WHOQOL group, 1998). The scale comprises 26 items across the following four domains: physical, psychological, social relationships and environment, including a facet of the overall QoL (general QoL and general health). Each subscale uses a 5-point response scale, and higher scores indicate greater QoL. This instrument has been validated for the Portuguese population according to the guidelines of the WHOQOL group and has demonstrated good reliability and 
validity (Vaz Serra et al., 2006). In this study, we excluded the environment domain of QoL. In this sample, Cronbach's alphas ranged from .72 (social QoL, G1) to .85 (psychological QoL, G1).

Intimacy. Marital intimacy was measured with the Personal Assessment of Intimacy in Relationships (PAIR; Schaefer \& Olson 1981). This self-report questionnaire contains 36 items, uses a 5-point Likert scale that ranges from 0 (strongly disagree) to 4 (strongly agree), and measures the degree of intimacy in a dyadic relationship (e.g., My partner listens to me when I need someone to talk to; My partner can really understand my hurts and joys). The total score for the scale ranges from 0 to 4 . In this study, the 6-item conventionality scale, which assesses the social desirability of individual responses, was not used. The psychometric properties of the Portuguese version (Moreira, Amaral, \& Canavarro, 2009) proved to be adequate, with a Cronbach's alpha of .92 for the total scale. In this study, we used the total score as a measure of global intimacy, and Cronbach's alphas were .93 (G1) and .89 (G2).

\section{Data Analysis}

All data analyses were conducted using the Statistical Package for the Social Sciences (SPSS, version 17.0; IBM SPSS, Chicago, IL). Missing data were random and low level $(<5 \%)$ and were handled by simple group mean substitution. Demographic and clinical data were not substituted. Descriptive statistics were computed for all sociodemographic, clinical and study variables. Before performing the main analysis, we explored the differences between the husbands whose wives were undergoing treatment and those whose wives were not receiving treatment with regard to the main study variables. In addition, to ensure the homogeneity of the groups, in terms of the main sociodemographic and clinical characteristics, differences between G1 and G2 were analyzed using chi-squared tests to compare the level of education, and univariate analyses of variance (ANOVAs) were used to compare age and the length of the relationships. 
An ANOVA was performed to test the differences between G1 and G2 in terms of intimacy, and a multivariate analysis of variance (MANOVA) of anxiety and depression symptomatology and of several domains of QoL was performed. When a multivariate effect was found, subsequent ANOVAs (one analysis per dependent variable) were performed. In order to explore the influence of women's treatment and surgery on their partners' adjustment, the group of cancer patients' husbands were divided into subgroups according to the treatment and surgery that their wives had received (radiotherapy and mastectomy; radiotherapy and conserving surgery; chemotherapy and mastectomy; and chemotherapy and conserving surgery). These four subgroups and the group of husbands of healthy women were compared in each analysis of variance. Therefore, a MANOVA of anxiety and depression symptomatology and of several domains of QoL was performed. When a multivariate effect was found, subsequent ANOVAs (one analysis per dependent variable) were performed. An ANOVA was performed to test the differences between groups in terms of intimacy. When a univariate effect was found, subsequent post-hoc Bonferroni tests were conducted in order to locate existing differences between groups.

The PROCESS computation tool (Hayes, 2012) was used to estimate six simple moderation models with the effect of intimacy on adjustment outcomes (depression and anxiety; psychological, physical, social and general QoL) moderated by the group type (G1 or G2). Prior to model estimation, intimacy was mean centered to reduce multicollinearity with the interaction term. In order to probe the significant interactions, the conditional effects (simple slopes) of intimacy on adjustment outcomes at the two values of the group type $(0=$ G2; $1=\mathrm{G} 1$ ) were generated. To graphically visualize the nature of the interaction, we used the estimates of the dependent variable (adjustment outcome) from the regression model for various values of intimacy and the two values of the moderator, generated by the PROCESS. 
Significance was set at the .05 level, and a partial eta squared $\left(\eta_{p}{ }^{2}\right)$ value provided the estimate of the effect size for the analyses of variance. Cohen (1988) has suggested that values of $.01, .06$ and .14 reflect small, medium and large effect sizes, respectively. Post-hoc power calculations were performed (G*Power; Faul, Erdfelder, Lang, \& Buchner, 2007). These calculations demonstrated that the statistical power $(.80)$ was sufficient to detect medium to large effects for all statistical analyses that were performed, given the sample size.

\section{Results}

\section{Preliminary Analyses}

Prior to conducting the main analyses, the differences between the husbands of women undergoing breast cancer treatments at the time of the study $(68.6 \%)$ and the husbands of women who had already completed their treatments (31.4\%) were analyzed. No significant differences were found in terms of intimacy, $F(1,64)=0.57, p=.545, \eta_{p}{ }^{2}=.01$, quality of life, Pillai's trace $=0.07, F(4,65)=1.29, p=.282, \eta_{p}{ }^{2}=.07$, or emotional adjustment, Pillai's trace $=0.02, F(2,67)=0.60, p=.550, \eta_{p}{ }^{2}=.02$. Therefore, all of the husbands of breast cancer patients, regardless of the treatment statuses of the women, were analyzed together in subsequent analyses.

Comparisons between G1 and G2 were performed for the main sociodemographic characteristics. These analyses showed that there were no significant differences between the groups with respect to age, $F(1,135)=3.87, p=.060, \eta_{p}{ }^{2}=.02$, length of marriage, $F(1,114)$ $=1.06, p=.306, \eta_{p}{ }^{2}=.01$, or education, $\chi 2(1, N=140)=0.41, p=.520$. Furthermore, bivariate relationships between the sociodemographic/clinical characteristics and outcome variables were examined to identify potential controls. No significant correlations were found among several sociodemographic and clinical variables (age, level of education, time from women's diagnosis to assessment, length of the relationship) and outcome variables. The 
exceptions were negative correlations between the physical QoL and age $(r=-.21, p=.014)$ and the length of marriage $(r=-.23, p=.014)$.

\section{Differences Between the Partners of Breast Cancer Patients and the Partners of Healthy}

\section{Women}

Table 1 shows the descriptive analyses for all of the study variables in G1, G2 and in the different subgroups, as well as the results of the comparison analyses.

We began by analyzing the differences between G1 (total group) and G2. The MANOVA for emotional adjustment revealed a significant multivariate effect, Pillai's Trace $=0.05, F(2,137)=3.54, p=.032, \eta_{p}{ }^{2}=.05$. The univariate $F$ tests indicated that the G1 husbands presented significantly more anxious, $F(1,138)=4.93, p=.028, \eta_{p}{ }^{2}=.03$, and depressive symptoms, $F(1,138)=6.13, p=.015, \eta_{p}{ }^{2}=.04$, than the $\mathrm{G} 2$ husbands (Table 1$)$.

With regard to the QoL domains, a significant multivariate effect was also found, Pillai's trace $=0.85, F(4,135)=3.12, p=.017, \eta_{p}^{2}=.09$. The subsequent univariate $F$ tests showed significant differences in general QoL, $F(1,138)=6.10, p=.015, \eta_{p}{ }^{2}=.04$, physical QoL, $F(1,138)=10.80, p=.001, \eta_{p}^{2}=.07$, and psychological QoL, $F(1,138)=10.37, p=$ $.002, \eta_{p}^{2}=.07$. The husbands of woman with breast cancer presented poor QoL in all of these domains (Table 1). No significant group differences were found in terms of social QoL, $F(1,138)=3.01, p=.081, \eta_{p}^{2}=.02$.

With regard to marital intimacy, a significant difference between G1 and G2 was found, $F(1,132)=9.86, p=.002, \eta_{p}^{2}=.07$, with the husbands of breast cancer patients reporting higher levels of intimacy in their relationship than the other men (Table 1).

We also explored the differences between the group of partners of healthy women and the different subgroups of the partners of breast cancer patients. The multivariate effect for emotional adjustment was not significant, Pillai's trace $=0.08, F(8,270)=1.45, p=.177, \eta_{p}{ }^{2}$ $=.04$. With regard to the QoL domains, a significant multivariate effect was found, Pillai's 
trace $=0.17, F(16,540)=1.92, p=.049, \eta_{p}{ }^{2}=.04$. The subsequent univariate $F$ tests showed significant differences in physical QoL, $F(4,135)=3.63, p=.008, \eta_{p}{ }^{2}=.10$, and psychological QoL, $F(4,135)=2.91, p=.024, \eta_{p}{ }^{2}=.08$. The subsequent post hoc tests showed no significant differences between the various treatment subgroups; the only significant difference was found between the husbands of healthy women and the husbands of patients who had performed chemotherapy and a mastectomy (subgroup C), with the latter group presenting poor physical and psychological QoL. No significant group differences were found in terms of general QoL, $F(4,135)=1.78, p=.137, \eta_{p}{ }^{2}=.05$, and social QoL, $F(4$, $135)=1.30, p=.275, \eta_{p}{ }^{2}=.04$

The ANOVA for marital intimacy revealed a significant difference between groups, $F(4,129)=3.14, p=.017, \eta_{p}^{2}=.09$. Post-hoc Bonferroni tests showed that the partners of healthy women reported less intimacy in their relationship than the husbands of women who had undergone chemotherapy, regardless of the type of surgery (subgroups C and D).

\section{The Association Between Intimacy and Emotional Adjustment and QoL: Does the}

\section{Experience of Cancer Moderate these Relationships?}

Table 2 presents the unstandardized coefficients and standard errors of the variables (independent variable, moderator and interaction) of each moderation model, as well as the model summary.

Emotional adjustment. The interaction between intimacy and depression $(b=-2.964$, $S E=0.969, p=.003$ ) explained a significant $5.3 \%$ of the additional variance in depressive symptomatology (Table 2). The conditional effect of intimacy on depression was significant for the G1 partners $(b=-3.65, t=-5.90, p<.001)$ but not for the G2 partners $(b=-0.71, t=-$ $0.95, p=.345)($ Figure 1).

For anxiety, the interaction between intimacy and group type was significant $(b=-$ $2.141, S E=1.076, p=.049)$ and explained $2.7 \%$ of the additional variance in anxiety (Table 
2). The conditional effect of intimacy on anxiety was only significant for the G1 partners, i.e., anxiety decreased as intimacy decreased only for the partners of breast cancer patients $(b=$ 2.23, $t=-3.24, p=.002)$. Among the partners of healthy woman, the slope did not differ significantly from zero, i.e., the conditional effect was not significant $(b=-0.09, t=-0.10, p=$ .918) (Figure 2).

Quality of life. The interaction between intimacy and group type was significant $(b=$ $13.068, S E=3.742, p<.001)$ and explained $6.4 \%$ of the total variance in psychological QoL (Table 2). As shown in Figure 3, there was a significant positive relationship between intimacy and psychological QoL among the partners of woman with breast cancer $(b=16.01$, $t=6.70, p<.001)$; however, this conditional effect was not significant for the partners of healthy women $(b=2.94, t=1.02, p=.309)$.

The interaction between intimacy and group type was also significant for social QoL $(b=12.639, S E=3.695, p<.001)$ and contributed to explaining $5.7 \%$ of the variance (Table 2). Simple slope analyses revealed that the partners who reported higher intimacy demonstrated greater social QoL both when their wives had breast cancer $(b=18.8, t=7.97$, $p<.001)$, and when their wives were healthy $(b=6.16, t=2.17, p=.032)$, although the slope was less pronounced (Figure 4).

No significant interactions were found for general $(b=6.845, S E=4.410, p=.123)$ and physical QoL $(b=4.729, S E=4.618, p=.308)$ (Table 2$)$. The effect of age was controlled in the physical QoL model $(b=-.018, p=.233)$ because of the significant correlation with this outcome variable. Although the length of marriage was also significantly correlated with physical QoL, this variable was excluded from use as a covariate to avoid a multicollinearity problem relating to the high correlation with age $(r=.74, p<.001)$.

\section{Discussion}


The main findings of this study were as follows: (1) the partners of breast cancer patients presented higher levels of emotional distress and poor QoL compared to the partners of healthy women; (2) the comparative analysis of the various subgroups revealed that the partners of women that had performed chemotherapy and a mastectomy presented lower levels of physical and psychological QoL compared to the partners of healthy women, and that the perception of intimacy was higher among the partners of patients who had undergone chemotherapy compared to the partners of healthy women; (3) higher levels of intimacy were related to lower levels of depression and anxiety and greater QoL only among the partners of breast cancer patients;

\section{The Partner Experience of Breast Cancer}

Psychosocial adjustment. The comparison of psychological adjustment between the partners of breast cancer patients and the partners of healthy women corroborated the first hypothesis of this study. As expected, a woman's cancer diagnosis has a major effect on her partner's adjustment, which was reflected as higher levels of anxious and depressive symptomatology and poorer QoL. This result is consistent with the study by Wagner et al. (2006), who also used a comparison group and found significant differences in QoL.

When the control group was compared to the four clinical subgroups, an interesting result emerged: the only significant difference was found between the control group and the partners of patients who performed chemotherapy and a mastectomy (in the physical and psychological QoL domains). Moreover, no differences were found between the different treatment/surgery subgroups. Given the small sample size of each subgroup, these analyses were merely exploratory and the results should be interpreted cautiously. It is strongly advised that future studies see the sample size of treatment subgroups increased, to ascertain whether these results are consistent. Despite the necessary precautions when interpreting the results, this data seems to suggest that the negative impact of the disease and treatment is particularly 
evident among the partners of patients who had undergone chemotherapy and performed a mastectomy. Although we are unaware of other studies that have examined the influence of surgery and treatment in a partner's adaptation, these results were, to some extent, expected. In fact, several studies have shown that the patients who receive chemotherapy usually present more adjustment difficulties than those who receive radiotherapy (So et al., 2010), due to the painful symptoms (e.g. nausea, fatigue) and body image changes (e.g., alopecia, weight changes) that often accompany this treatment. The side effects of the radiation therapy (e.g., skin reactions, fatigue) are usually less severe and demanding, from a psychological and physical point of view. Therefore, it is likely that the patients who had undergone chemotherapy felt more distressed and this impacted on their partners' QoL. Because of the interdependence that characterizes a couple, we may hypothesize that the eventual distress that was experienced by these patients may have had a negative effect on their partners' physical and psychological QoL. Although this study has not assessed the adjustment of women (and therefore this explanation is merely hypothetical), previous studies have proven that there is a strong effect on partners and that distress levels may be positively correlated (Northouse et al., 2001; Segrin et al., 2007). In addition, the caregiver's tasks associated with chemotherapy are likely to be more demanding than those associated with radiotherapy (e.g., the partner may have to assume many of the women's roles, accompany his wife to treatment), which in turn may negatively interfere with their physical and psychological QoL.

It is also important to note that these women had undergone a mastectomy, which could be more distressing to the partner than a conserving surgery. In their literature review, Carlson et al. (2000) indicated that although over time the partners and the patients adjust well to this surgery, initially, this procedure causes more distress to the partners than a breast conserving surgery does. 
Our results have also shown that when assessed together, the group of breast cancer patients' partners presented poor QoL and higher levels of depressive and anxious symptomatology. In order to better understand these findings, it is important to consider that the partner is often the main informal caregiver of the patient. Considering that breast cancer is a chronic disease that may require long-term treatment and assistance, a partner may need to perform the caregiver role for a long time. This responsibility may lead a partner to feel increasingly burdened by the demands that are associated with this role, such as assistance with medical care, the provision of emotional support, and financial management. In addition, the provision of care may prevent a partner from satisfying his own social, emotional and/or supportive needs and result in an increase in stress related to these caregiving tasks. The caregiver burden may explain the poorer adjustment that was observed among the partners of breast cancer patients when compared with the spouses of healthy women (Nijboer et al., 1998). This explanation is consistent with the findings of previous studies. For instance, Braun et al. (2007) found that one of the main predictors of depression in partners of patients with breast cancer was the subjective caregiving burden (i.e., the caregivers' assessment of their role as caregivers or their appraisal of the difficulty of their caregiving tasks).

Marital intimacy. Breast cancer may influence both the psychosocial adjustment and marital relationships of partners. The results of this study showed that the partners of women with breast cancer perceived their relationship as more intimate or close as compared with the spouses of healthy women.

In addition, the comparison of the different treatment subgroups showed that the partners of patients who had undergone chemotherapy reported higher levels of intimacy than the control group. These results, however needing to be cautiously interpreted, seem to indicate that the experience of chemotherapy promotes the perception of intimacy, regardless of the type of surgery. In fact, by them dealing together with the demands and difficulties 
associated with this aversive treatment, they may increase the proximity felt between them as a couple. Although there were no differences between the various clinical subgroups, the patients' partners who performed radiotherapy did not differ significantly from the husbands of healthy women. This may be due to a lower need of patient assistance and to a lower perception of the severity of the disease as the treatment is not as demanding as chemotherapy. It can thus be hypothesized that the need to provide greater support to the patients who undergo chemotherapy, although it can lead to an increased burden, can also lead to an increased involvement by the partner, and to a greater sharing and communication between the couple. This, in turn, may then lead to a greater perception of intimacy by the partner.

The increased perception of intimacy is consistent with several studies that have demonstrated that marital relationships are challenged but not necessarily harmed by the disease and that a frequent result of this experience is the strengthening of this relationship. In fact, research has revealed that most couples adapt well and have sufficient resources to cope with illness (Dorval et al., 2005; Taylor-Brown, Kilpatrick, Maunsell, \& Dorval, 2000). Most couples do not report a decrease in the quality of their marital relationship (Manne, 1998), and only a small proportion of couples experience difficulties or separation following diagnosis (Dorval, Maunsell, Taylor-Brown, \& Kilpatrick, 1999; Taylor-Brown et al., 2000). In addition, many couples report a strengthening of their relationship (Dorval et al., 2005; Hinnen et al., 2008) and the occurrence of positive changes or relational growth after this experience (Weiss, 2004). Therefore, the perception of increased marital intimacy among the spouses of cancer patients may reflect this general process of relationship enhancement that is observed among many couples. Future longitudinal studies should assess the perceived marital intimacy of partners before and after the diagnosis to enable more robust conclusions 
regarding this eventual strengthening of the relationship and the supposed increase in intimacy.

\section{The Influence of Marital Intimacy on the Psychosocial Adjustment of Partners}

Our results showed that an increase in marital intimacy was associated with an increase in psychological and social QoL and a decrease in levels of depressive and anxious symptomatology among the partners of breast cancer patients. These findings confirm our hypothesis and are consistent with Manne and Badr's model (2008), according to which intimacy is a primary mechanism for promoting couples' psychological adjustment to a cancer disease. Similar findings were obtained by Manne and Badr (2010) in a study of head and neck and lung cancer patients and their partners. Although the study did not involve a comparison group of spouses of healthy women, the authors found that partners who reported higher levels of intimacy reported almost no distress in contrast with those who reported lower levels of intimacy (these associations were stronger among partners than among patients). However, it is important to note that approximately $66 \%$ of the partners in that study were female in contrast with the sample in the current study.

Although the literature has consistently shown that marital intimacy has beneficial effects on individual health and well-being (Prager, 1995; Prager \& Buhrmester, 1998) regardless of the presence or absence of a stressful life situation, a moderating effect of the group was found: the associations between marital intimacy and psychosocial adjustment were significant only among the partners of breast cancer patients (with the exception of social QoL, which was also related to intimacy among the partners of healthy woman, although the regression slope was much less pronounced). These results can be explained in the context of a life-threatening disease because intimate relationships, such as the marital relationship, often assume a more powerful role in determining individual well-being than in situations that lack such a threat (Bolger, Foster, Vinokur, \& Ng, 1996). The threat of losing 
one's partner may have triggered the partner's attachment system and thus increased the desire for closeness or intimacy. As discussed, higher levels of intimacy were found among the partners of cancer patients. According to attachment theory, in periods of threat and distress, the need for closeness and support from significant others increases (Collins \& Feeney, 2004). This need could explain why marital intimacy was more important for the partners of cancer patients than for the partners of healthy women. In addition, the greater perception of intimacy among the partners of cancer patients could also have contributed to the stronger influence on their adjustment outcomes.

\section{Strengths and Limitations}

One of the main contributions of this study is the focus on the partners of patients. Research has primarily focused on the experience of women and their own adjustment to breast cancer, and studies pertaining to the adjustment of partners are clearly insufficient. Moreover, the few studies that included partners examined their psychosocial adjustment in comparison with the adjustment of women, which would not provide an accurate evaluation of the adjustment of the male partners. The inclusion of a comparison group is an additional strength of this study, as this comparison enabled the assessment of the specific effect of the experience of a woman's cancer on her partner's emotional distress and QoL. Another important contribution was the adoption of a comprehensive approach to the adjustment of partners by including both emotional distress (anxiety and depression symptomatology) and a positive dimension of adjustment (QoL). This approach emphasizes the importance of adopting a non-deterministic and non-pathologic perspective in both research and practice. Finally, the study of the influence of marital intimacy on the QoL and emotional distress of partners is also an important and innovative contribution. To our knowledge, no study has investigated the role of this important marital dimension among a sample of spouses of breast cancer patients. 
Some limitations should also be mentioned. The cross-sectional design of this study does not allow for causal inference. Therefore, the links between the variables could be bidirectional (e.g., higher levels of intimacy may promote greater QoL, but greater QoL may also promote higher levels of intimacy). Future work should involve longitudinal designs to better understand the links between these variables over time. Second, the small sample size decreases the statistical power that is necessary to detect differences with a small effect size. For instance, the power analyses for the ANOVAs and MANOVAs revealed that only medium to large effects could be detected and that small effects could have been missed. It should also be noted that the sample size of each subgroup of cancer patients' partners was very small and, therefore, the comparison analyses of these groups was only exploratory. Future studies with larger samples are necessary to examine the same mean differences and moderation effects. Finally, the group of partners of breast cancer patients was quite heterogeneous with respect to the time that had elapsed since diagnosis (from 1 to 60 months post-diagnosis). Although no differences were found between the partners whose wives were undergoing treatment and those whose treatment was already completed, two different disease phases were analyzed. Futures studies should ideally examine the psychosocial adjustment of partners in specific phases of cancer (from diagnosis to survival) in a longitudinal manner.

\section{Practical Implications}

The partner of a breast cancer patient is often a hidden patient. Given the evident effect of the disease on the adaptation and well-being of women and the need to implement strategies that promote their well-being and psychological adjustment, the support needs of partners are often neglected. Simultaneously, the caregiver role that partners often assume may prevent them from expressing their own needs for support in an attempt to more effectively assist the patient. Thus, not only are professionals more attentive to women and favor interventions that are specifically directed toward women, but the partners can also hide 
their emotional states and thus fail to express any need for psychotherapeutic assistance. The results of this study clearly showed that partners are also affected by the disease. The significantly higher levels of anxiety and depression indicate that partners may need and benefit from psychotherapeutic support. Thus, healthcare professionals who follow these women should be equally attentive to their spouses by providing the necessary conditions to ensure that their needs are met.

In addition, our findings suggest that promoting intimacy between spouses may have a positive effect on the adjustment of partners. Therefore, strategies that aim to enhance the closeness between spouses should be considered. Given the critical role of self-disclosure in establishing and maintaining intimacy (Laurenceau et al., 2004; Manne \& Badr, 2008, 2010; Reis \& Shaver, 1988), a possible avenue for intervention would be the implementation of strategies that aim to encourage open communication between patients and their partner and the free expression of feelings and thoughts. For instance, Manne and Ostroff (2008) developed a couple-focused program directed toward patients with breast cancer and their partners with the purpose of promoting the well-being of both partners. This intervention is based on cognitive-social processing theory and cognitive-behavioral theories and emphasizes, among other aspects, the importance of sharing thoughts and feelings related to cancer to facilitate the cognitive processing of this event. 


\section{References}

Bolger, N., Foster, M., Vinokur, A., \& Ng, R. (1996). Close relationships and adjustment to a life crisis: The case of breast cancer. Journal of Personality and Social Psychology, 70(2), 283-294.

Braun, M., Mikulincer, M., Rydall, A., Walsh, A., \& Rodin, G. (2007). Hidden Morbidity in Cancer: Spouse Caregivers. Journal of Clinical Oncology, 25(30), 4829-4834.

Broderick, C. (1993). Understanding family processes: Basics of family systems therapy. Thousand Oaks, CA: Sage Publications, Inc.

Broucke, S., Vandereycken, W., \& Vertommen, H. (1995). Marital intimacy: Conceptualization and assessment. Clinical Psychology Review, 15(3), 217-233.

Carlson, L.E., Bultz, B.D., Speca, M., \& Pierre, M. (2000). Partners of cancer patients: Part I - Impact, adjustment, and coping across the illness trajectory. Journal of Psychosocial Oncology, 18(2), 39-63.

Cohen, J. (1988). Statistical power analysis for the behavioural sciences (2nd ed.). Hillsdale: Erlbaum.

Collins, N.L., \& Feeney, B.C. (2004). An attachment theory perspective on closeness and intimacy. In D. J. Mashek \& A. Aron (Eds.), Handbook of closeness and intimacy. New Jersey \& London: Lawrence Erlbaum.

Dorros, S., Card, N., Segrin, C., \& Badger, T. (2010). Interdependence in women with breast cancer and their partners: An interindividual model of distress. Journal of Consulting and Clinical Psychology, 78(1), 121-125.

Dorval, M., Guay, S., Mondor, M., Mâsse, B., Falardeau, M., Robidoux, A., ... Maunsell, E. (2005). Couples who get closer after breast cancer: Frequency and predictors in a prospective investigation. Journal of Clinical Oncology, 23(15), 3588-3596. 
Dorval, M., Maunsell, E., Taylor-Brown, J., \& Kilpatrick, M. (1999). Marital stability after breast cancer. Journal of the National Cancer Institute, 91, 54-59.

Faul, F., Erdfelder, E., Lang, A.-G., \& Buchner, A. (2007). G*Power 3: A flexible statistical power analysis program for the social, behavioural, and biomedical sciences. Behaviour Research Methods, 39, 175-191.

Greeff, A., \& Malherbe, H. (2001). Intimacy and marital satisfaction in spouses. Journal of Sex \& Marital Therapy, 27, 247-257.

Hagedoorn, M., Sanderman, R., Coyne, J., Bolks, H., \& Tuinstra, J. (2008). Distress in couples coping with cancer: A meta-analysis and critical review of role and gender effects. Psychological Bulletin, 134(1), 1-30.

Hayes, A. F. (2012). PROCESS: A versatile computational tool for observed variable mediation, moderation, and conditional process modeling [White paper]. Retrieved from http://www.afhayes.com/public/process2012.pdf

Hinnen, C., Ranchor, A., Sanderman, R., Snijders, T., Hagedoorn, M., \& Coyne, J. (2008). Course of distress in breast cancer patients, their partners, and matched control couples. Annals of Behavioral Medicine, 36(2), 141-148.

Kayser, K., \& Scott, J. (2008). Helping couples cope with women's cancers: An evidencebased approach for practitioners. New York: Springer.

Kim, Y. \& Given, B. (2008). Quality of life of family caregivers of cancer survivors across the trajectory of the illness. Cancer, 112(11 suppl), 2556-2568.

Laurenceau, J.-P., Barrett, L., \& Pietromonaco, P. (2004). Intimacy as an interpersonal process: The importance of self-disclosure, partner disclosure, and perceived partner responsiveness in interpersonal exchanges. In H.T. Reis \& C.E. Rusbult (Eds.), Close Relationships (pp. 199-211). New York: Taylor \& Francis Books, Inc. 
Manne, S. (1998). Cancer in the marital context: A review of the literature. Cancer Investigation, 16(3), 188-202.

Manne, S., Alfieri, T., Taylor, K., \& Dougherty, J. (1999). Spousal negative responses to cancer patients: The role of social restriction, spouse mood, and relationship satisfaction. Journal of Consulting and Clinical Psychology, 67(3), 352-361.

Manne, S., \& Badr, H. (2008). Intimacy and relationship processes in couples' psychosocial adaptation to cancer. Cancer, 112(11 suppl), 2541-2555.

Manne, S., \& Badr, H. (2010). Intimacy processes and psychological distress among couples coping with head and neck or lung cancers. Psycho-Oncology, 19(9), 941-954.

Manne, S., Badr, H., Zaider, T., Nelson, D., \& Kissane, D. (2010). Cancer-related communication, relationship intimacy, and psychological distress among couples coping with localized prostate cancer. Journal of Cancer Survivorship, 4(1), 74-85.

Manne, S. \& Ostroff, J. (2008). Coping with breast cancer: A couples-focused group intervention. New York, NY: Oxford University Press.

Moreira, H., Crespo, C., Paredes, T., Silva, S., Canavarro, M. C. \& Dattilio, F. (2011). Marital relationship, body image and quality of life among breast cancer patients at the treatment and survivorship phases. Journal of Contemporary Family Therapy, 33(2), $161-178$

Moreira, H., Amaral, A., \& Canavarro, M.C. (2009). Adaptação do Personal Assessment of Intimacy in Relationships Scale (PAIR) para a população Portuguesa: Estudo das suas características psicométricas [Adaptation of the Personal Assessment of Intimacy in Relationships Scale (PAIR) for the Portuguese population: Study of its psychometric properties]. Psychologica, 50, 353-373. 
Nakaya, N., Saito-Nakaya, K., Bidstrup, P., Dalton, S., Frederiksen, K., Steding-Jessen, M., ... Johansen, C. (2010). Increased risk of severe depression in male partners of women with breast cancer. Cancer, 116, 5527-5534.

Nijboer, C., Tempelaar, R., Sanderman, R., Triemstra, M., Spruijt, R., \& Van den Bos, G. (1998). Cancer and caregiving: The impact on the caregiver's health. PsychoOncology, 7, 3-13.

Northouse, L., Templin, T., \& Mood, D. (2001). Couples' adjustment to breast disease during the first year following diagnosis. Journal of Behavioral Medicine, 24(2), 115-136.

Northouse, L., Templin, T., Mood, D., \& Oberst, M. (1998). Couples' adjustment to breast cancer and benign breast disease: A longitudinal analysis. Psycho-Oncology, 7, 37-48.

O'Mahoney, J., \& Carroll, R. (1997). The impact of breast cancer and its treatment on marital functioning. Journal of Clinical Psychology in Medical Settings, 4(4), 397-414.

Pais-Ribeiro, J., Silva, I., Ferreira, T., Martins, A., Meneses, R., \& Baltar, M. (2007).

Validation Study of a Portuguese version of the hospital anxiety and depression scale. Psychology, Health \& Medicine, 12(2), 225-235.

Prager, K.J. (1995). The Psychology of Intimacy. New York: The Guilford Press.

Prager, K.J., \& Buhrmester, D. (1998). Intimacy and need fulfilment in couple relationship. Journal of Social and Personal Relationships, 15, 435-469.

Reis, H.T., \& Patrick, B. (1996). Attachment and Intimacy: Component Processes. In T. Higgins \& A. Kruglanski (Eds.), Social Psychology: Handbook of Basic Principles. New York: The Guilford Press.

Reis, H.T., \& Shaver, P. (1988). Intimacy as an interpersonal process. In S. Duck (Ed.), Handbook of personal relationships (pp. 367-389). Chichester: Wiley.

Schaefer, M., \& Olson, D. (1981). Assessing intimacy: The PAIR Inventory. Journal of Marital and Family Therapy, 7(1), 47-60. 
Segrin, C., Badger, T., Dorros, S., Meek, P., \& Lopez, A. (2007). Interdependent anxiety and psychological distress in women with breast cancer and their partners. PsychoOncology, 16, 634-643.

Sjövall, K., Attner, B., Lithman, T., Noreen, D., Gunnars, B., Thomé, B., \& Olsson, H. (2009). Influence on the health of the partner affected by tumor disease in the wife or husband based on a population-based register study of cancer in Sweden. Journal of Clinical Oncology, 27, 4781-4786.

So, W., Marsh, G., Ling, W., Leung, F., Lo, J., Yeung, M., \& Li, G. (2010). Anxiety, depression and quality of life among Chinese breast cancer patients during adjuvant treatment. European Journal of Oncology Nursing, 14(1), 17-22.

Taylor-Brown, J., Kilpatrick, M., Maunsell, E., \& Dorval, M. (2000). Partner abandonment of women with breast cancer: Myth or reality? Cancer Practice, 8(4), 160-164.

Vaz Serra, A., Canavarro, M.C., Simões, M., Pereira, M., Gameiro, S., Quartilho, M., et al. (2006). Estudos psicométricos do instrumento de Avaliação da Qualidade de Vida da Organização Mundial de Saúde (WHOQOL-bref) para Português de Portugal.

[Psychometric studies of the World Health Organization Quality of Life Assessment (WHOQOL-Bref) for Portuguese from Portugal]. Psiquiatria Clínica, 27(1), 41-49.

Wagner, C., Bigatti, S., \& Storniolo, A. (2006). Quality of life of husbands of women with breast cancer. Psycho-Oncology, 15, 109-120.

Weiss, T. (2004). Correlates of posttraumatic growth in husbands of breast cancer survivors. Psycho-Oncology, 13, 260-268.

Whoqol-Group. (1998). Development of World Health Organization WHOQOL-bref Quality of Life Assessment. Psychological Medicine, 28, 551-558.

Zigmond, A.P., \& Snaith, R.P. (1983). The Hospital and Depression Scale. Acta Psychiatrica Scandinavica, 67, 361-370. 


\section{Table 1}

Descriptive and comparison analyses

\begin{tabular}{|c|c|c|c|c|c|c|c|c|c|c|c|}
\hline \multirow{3}{*}{$\begin{array}{l}\text { Dependent } \\
\text { Variable }\end{array}$} & \multirow{3}{*}{$\begin{array}{c}\text { G2 - Controls } \\
M(D P) \\
(n=70)\end{array}$} & \multicolumn{10}{|c|}{ Partners of breast cancer patients $(n=70)$} \\
\hline & & \multirow{2}{*}{$\begin{array}{l}\text { G1 - Total } \\
\text { group } \\
M(D P) \\
(n=70)\end{array}$} & \multicolumn{2}{|c|}{$\begin{array}{c}\text { Group } \\
\text { difference }^{\text {a }}\end{array}$} & \multirow{2}{*}{$\begin{array}{c}\mathbf{A}- \\
\text { Radiotherapy } \\
\text { and } \\
\text { Mastectomy } \\
M(D P) \\
(n=20)\end{array}$} & \multirow{2}{*}{$\begin{array}{c}\mathbf{B}- \\
\text { Radiotherapy } \\
\text { and } \\
\text { Conservative } \\
\text { Surgery } \\
M(D P) \\
(n=8) \\
\end{array}$} & \multirow{2}{*}{$\begin{array}{c}\mathbf{C}- \\
\text { Chemotherapy } \\
\text { and } \\
\text { Mastectomy } \\
M(D P) \\
(n=24)\end{array}$} & \multirow{2}{*}{$\begin{array}{c}\mathbf{D}- \\
\text { Chemotherapy } \\
\text { and } \\
\text { Conservative } \\
\text { Surgery } \\
M(D P) \\
(n=18) \\
\end{array}$} & \multicolumn{2}{|c|}{$\begin{array}{c}\text { Group } \\
\text { difference }^{\mathrm{b}}\end{array}$} & \multirow[t]{2}{*}{$\begin{array}{c}\text { Pairwise } \\
\text { comparisons }\end{array}$} \\
\hline & & & $F$ & $p$ & & & & & $F$ & $p$ & \\
\hline \multicolumn{12}{|c|}{ Emotional Adjustment } \\
\hline Anxiety & $6.00(2.78)$ & $7.21(3.64)$ & 4.93 & .028 & $6.70(3.50)$ & $6.88(2.17)$ & $7.50(4.42)$ & $7.56(3.31)$ & 1.46 & .22 & --- \\
\hline Depression & $3.47(2.89)$ & $4.81(3.50)$ & 6.13 & .015 & $5.00(3.96)$ & $3.00(3.38)$ & $5.08(3.45)$ & $5.05(3.11)$ & 2.25 & .67 & --- \\
\hline \multicolumn{12}{|l|}{ QoL } \\
\hline General & $71.25(11.72)$ & $65.54(15.41)$ & 6.10 & .015 & $66.62(15.64)$ & $65.63(17.36)$ & $63.54(17.26)$ & $68.06(12.94)$ & 1.78 & .137 & --- \\
\hline Physical & $78.06(12.04)$ & $70.20(15.97)$ & 10.80 & .001 & $72.62(19.47)$ & $75.00(13.63)$ & $65.71(14.07)$ & $69.84(13.48)$ & 3.63 & .008 & $\mathrm{G} 2 \neq \mathrm{C}$ \\
\hline Psychological & $78.15(8.58)$ & $71.25(15.75)$ & 10.37 & .002 & $71.88(14.45)$ & $75.00(13.36)$ & $68.96(20.83)$ & $71.30(12.37)$ & 2.91 & .024 & $\mathrm{G} 2 \neq \mathrm{C}$ \\
\hline Social & $73.57(11.26)$ & $69.52(15.60)$ & 3.01 & .081 & $66.67(21.80)$ & $66.67(7.72)$ & $71.88(14.29)$ & $70.83(11.52)$ & 1.30 & .275 & --- \\
\hline Intimacy & $2.44(0.47)$ & $2.73(0.58)$ & 9.86 & .002 & $2.57(0.71)$ & $2.63(0.35)$ & $2.80(0.59)$ & $2.82(0.50)$ & 3.14 & .017 & $\begin{array}{c}\mathrm{G} 2 \neq \mathrm{C} ; \mathrm{G} 2 \neq \\
\mathrm{D}\end{array}$ \\
\hline
\end{tabular}

Note. ${ }^{a}$ Univariate Analyses of Variance (including groups G1 and G2); ${ }^{b}$ Univariate Analyses of Variance (including groups G2 and A to D) 


\section{Table 2}

Moderation analyses

\section{Dependent variables}

\begin{tabular}{|c|c|c|c|c|c|c|c|c|c|c|c|c|}
\hline \multirow[b]{2}{*}{ Intimacy } & \multicolumn{2}{|c|}{ Depression } & \multicolumn{2}{|c|}{ Anxiety } & \multicolumn{2}{|c|}{ Psychological QoL } & \multicolumn{2}{|c|}{ Social QoL } & \multicolumn{2}{|c|}{ Physical QoL } & \multicolumn{2}{|c|}{ General QoL } \\
\hline & $b$ & $S E$ & $b$ & $S E$ & $b$ & $S E$ & $b$ & $S E$ & $b$ & $S E$ & $b$ & $S E$ \\
\hline Group $^{a}$ & $1.977 * * *$ & 0.515 & $1.502 * *$ & 0.571 & $-9.705 * * *$ & 1.987 & $-8.786^{* * *}$ & 1.962 & $-8.963 * * *$ & 2.525 & $-8.364 * * *$ & 2.342 \\
\hline Intimacy & -0.707 & 0.746 & -0.085 & 0.828 & 2.942 & 2.880 & $6.161 *$ & 2.844 & 3.467 & 3.602 & 2.876 & 3.394 \\
\hline Group x Intimacy & $-2.964 * *$ & 0.969 & $-2.141^{*}$ & 1.076 & $13.068 * * *$ & 3.742 & $12.639 * * *$ & 3.695 & 4.729 & 4.618 & 6.845 & 4.410 \\
\hline Total $R^{2}$ & \multicolumn{2}{|c|}{.249} & \multicolumn{2}{|c|}{.104} & \multicolumn{2}{|c|}{.313} & \multicolumn{2}{|c|}{.368} & \multicolumn{2}{|c|}{.150} & \multicolumn{2}{|c|}{.140} \\
\hline$F($ final model $)$ & \multicolumn{2}{|c|}{$14.38 * * *$} & \multicolumn{2}{|c|}{$5.007 * *$} & \multicolumn{2}{|c|}{$19.74 * * *$} & \multicolumn{2}{|c|}{$25.26 * * *$} & \multicolumn{2}{|c|}{$5.60 * * *$} & \multicolumn{2}{|c|}{$7.03 * * *$} \\
\hline
\end{tabular}

Note. $\mathrm{b}=$ unstandardized coefficient, $\mathrm{SE}=$ standard error. Age was introduced as a covariate in the model of physical QoL $(b=-0.18, S E=0.153, p=.233)$.

${ }^{\mathrm{a}} 0=$ Partners of women without cancer history (G2); 1 = Partners of breast cancer patients (G1)

${ }^{*} p<.05 .{ }^{* *} p<.01 .{ }^{* * *} p<.001$. 
Figure 1. The moderating effect of group type on the association between inti depression

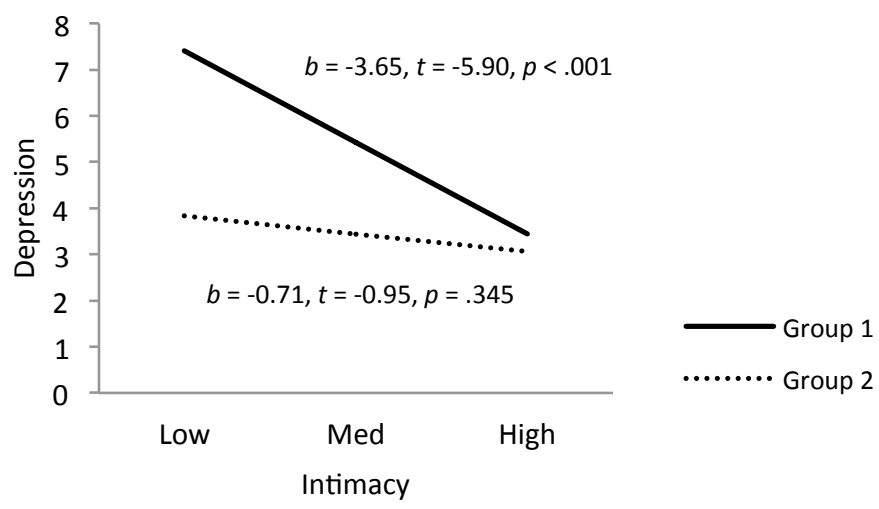


Figure 2. The moderating effect of group type on the association between intimacy and anxiety

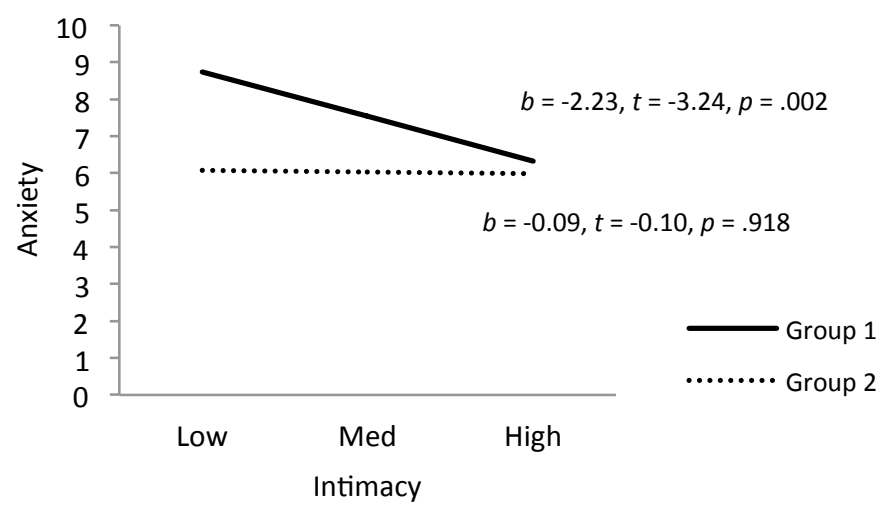


Figure 3. The moderating effect of group type on the association between intimacy and psychological QoL

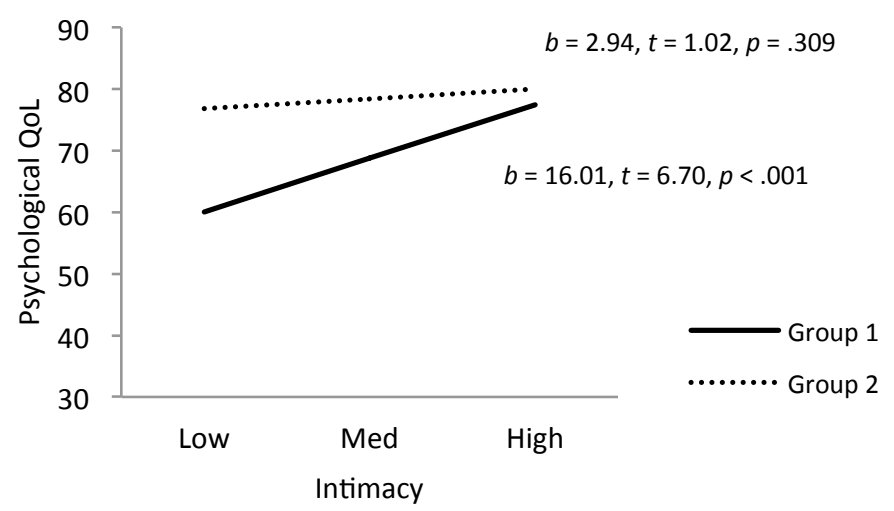


Figure 4. The moderating effect of group type on the association between intimacy and social QoL

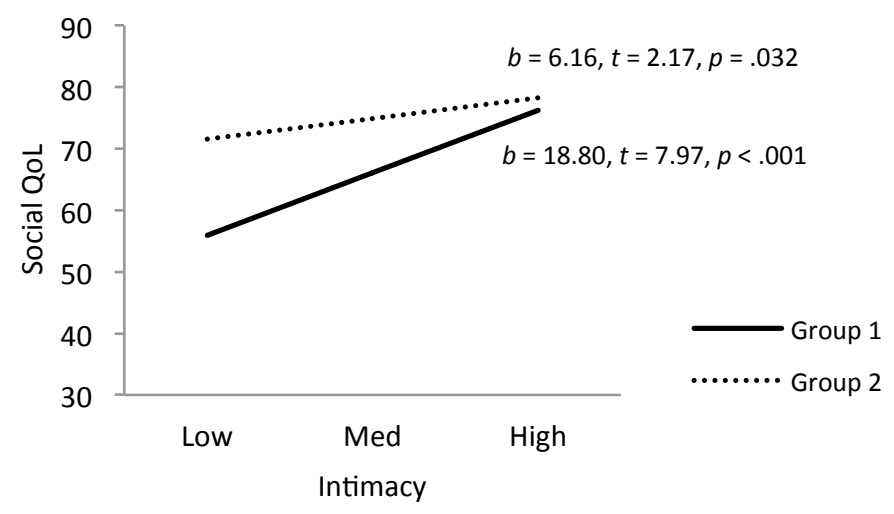

